\title{
Metodologia de Projeto e Implementação em DSP de Detectores Multiusuário Heurísticos DS/CDMA
}

\section{Methodology and Implementation on DSP of Heuristic Multiuser DS/CDMA Detectors}

\author{
Alex Miyamoto Mussi ${ }^{1}$; Rafael de Oliveira Ribeiro ${ }^{1}$; Taufik Abrão ${ }^{2}$
}

\section{Resumo}

O crescente aumento do número de usuários de redes de comunicações móveis e a escassez do espectro eletromagnético fazem com que o emprego de técnicas de diversidade e a detecção/ decodificação eficientes, tais como o emprego de múltiplas antenas no transmissor e/ou no receptor, detecção multiusuário (MuD - Multiuser Detection), entre outras, tenham um papel cada vez mais preponderante no cenário das telecomunicações. Este trabalho apresenta uma metodologia de projeto baseada em processadores digitais de sinais (DSP - Digital Signal Processor), tendo em vista a implementação de detectores multiusuário heurísticos em sistemas DS/CDMA (Direct Sequence Code Division Multiple Access). Técnicas de detecção heurísticas resultam em desempenho quase-ótimos, no sentido de se aproximarem do desempenho de máxima-verossimilhança (ML). Neste trabalho, empregou-se plataforma de desenvolvimento em DSP denominada DSK C6713, a qual está baseada no processador Texas TMS320C6713. As técnicas heurísticas implementadas baseiam-se em algoritmos bem estabelecidos na literatura. A eficiência dos algoritmos implementados em DSP foi avaliada numericamente via cômputo da medida da taxa de erro de bit (BER). Finalmente, a viabilidade da implementação em DSP pôde então ser comprovada através da comparação de vários resultados de simulação Monte-Carlo em MatLab, com aqueles obtidos diretamente da implementação em DSP. Demonstra-se ainda o efetivo aumento de desempenho e de capacidade do sistema DS/CDMA com a utilização de técnicas de detecção heurística multiusuário, implementadas diretamente em DSP.

Palavras-chave: Telefonia celular. CDMA. Detecção multiusuário. Algoritmos heurísticos. Processadores digitais de sinais. Complexidade de implementação.

\begin{abstract}
The growing number of users of mobile communications networks and the scarcity of the electromagnetic spectrum make the use of diversity techniques and detection/decoding efficient, such as the use of multiple antennas at the transmitter and/or receiver, multiuser detection (MuD - Multiuser Detection), among others, have an increasingly prominent role in the telecommunications landscape. This paper presents a design methodology based on digital signal processors (DSP - Digital Signal Processor) with a view to the implementation of multiuser heuristics detectors in systems DS/CDMA (Direct Sequence Code Division Multiple Access). Heuristics detection techniques result in near-optimal performance in order to approach the performance of maximum-likelihood (ML). In this work, was employed the DSP development platform called the C6713 DSK, which is based in Texas TMS320C6713 processor. The heuristics techniques proposed are based on well established algorithms in the literature. The efficiency of the algorithms implemented in DSP has been evaluated numerically by computing the measure of bit
\end{abstract}

\footnotetext{
Alunos de mestrado do Departamento de Engenharia Elétrica, Universidade Estadual de Londrina.

2 Docente do Departamento de Engenharia Elétrica da Universidade Estadual de Londrina (DEEL-UEL). E-mail: taufik@uel.br
} 
error rate (BER). Finally, the feasibility of implementation in DSP could then be verified by comparing results from multiple Monte-Carlo simulation in Matlab, with those obtained from implementation on DSP. It also demonstrates the effective increase in performance and system capacity of DS/CDMA with the use of heuristic multiuser detection techniques, implemented directly in the DSP.

Key words: Mobile communication. CDMA. Multiuser detection. Heuristic algorithms. Digital signal processors. Complexity.

\section{Introdução}

A técnica CDMA é caracterizada pelo compartilhamento do mesmo recurso espectral por todos os usuários ativos, ou seja, os usuários transmitem simultaneamente em uma mesma banda de frequência. Para isso, a informação de cada usuário é multiplicada por uma sequência, chamada código de espalhamento, que ocupa uma banda muito maior que a da informação, fazendo com que a informação de cada usuário, agora "espalhada" espectralmente, ocupe uma banda muito maior que a mínima necessária; isto é chamado de espalhamento espectral (SS - Spread Spectrum). Cada usuário possui seu próprio código de espalhamento, de forma que se tenha uma situação onde ou haja ortogonalidade entre os códigos ou, de forma ainda favorável, as correlações (ou interferência de múltiplo acesso) entre os sinais dos usuários sejam reduzidas. No receptor, o sinal é "desespalhado" via multiplicação por uma réplica do código de cada usuário e, devido à baixa correlação entre cada código (ou virtualmente é zero ou mesmo existe ortogonalidade entre eles), a informação dos outros usuários são interpretadas como ruído, chamado de interferência de múltiplo acesso (MAI - Multiple Access Interference).

Em sistemas DS-CDMA existem duas maneiras para a detecção da informação, a convencional ( $\mathrm{SuD}$ - Single-user Detector) e a multiusuário (MuD Multiuser Dectetor).

$\mathrm{Na}$ detecção convencional cada ramo detecta um único usuário sem levar em consideração a existência dos demais, geralmente utilizando-se de um banco de filtros casados (MFB - Matched Filter Bank), ou receptor de correlação. Para modulação BPSK (Binary Phase Shift Keying), este filtro, casado ao código de espalhamento e a formação de pulso/ chip, é composto de um integrador seguido de um amostrador-retentor e um circuito final de decisão. Não ocorre compartilhamento da informação nem processamento dos sinais dos demais usuários ativos. Neste contexto, a interferência MAI é tratada simplesmente como ruído de fundo (AWGN Additive White Gaussian Noise).

$\mathrm{Na}$ detecção $\mathrm{MuD}$, informações dos usuários ativos no sistema são utilizadas conjuntamente a fim de atenuar/eliminar a interferência provocada entre si para melhor detectar cada usuário individualmente, aumentando o desempenho e/ou capacidade do sistema móvel celular de modo a aproximá-la da capacidade do canal (ABRÃO, 2001).

Em sistemas DS/CDMA, a limitação de desempenho e capacidade do sistema é resultado principalmente da MAI. Ela torna-se substancial quando o número de usuários cresce e/ou quando as disparidades de potência crescem. Assim, enquanto a detecção convencional considera todos os usuários interferentes como ruído, a detecção multiusuário constitui uma melhor estratégia justamente por utilizar as informações destes usuários no processo de detecção, combatendo efetivamente a interferência de múltiplo acesso, resultando em melhoria de desempenho em relação ao detector convencional (VERDÚ, 1986).

Entre as técnicas de detecção $\mathrm{MuD}$, o melhor desempenho possível é obtido com o detector ótimo (OMuD) de máxima verossimilhaça (ML-Maximum Likelihood), porém às custas de uma complexidade computacional que cresce exponencialmente com o número de usuários, tornando-o inviável, atualmente, para implementação em sistemas de interesse comercial (VERDÚ, 1998). 
Devido à alta complexidade do $\mathrm{OMuD}$ (Optimum $M u D)$, inúmeros detectores multiusuários foram desenvolvidos visando a redução de complexidade com a manutenção de um certo desempenho. Detectores que atendem a este critério são denominados MuDs sub-óptimos, entre estes destacam-se os lineares MMSE (Minimum Mean Squared Error) e o Decorrelacionador, além dos $\mathrm{MuDs}$ não lineares sub-ótimos PIC (Parallel Interference Cancelation), SIC (Successive Interference Cancelation) e ZF-DF (Zero Forcing Decision Feedback) (ABRÃO, 2001; VERDÚ, 1998).

Alternativamente, na última década, técnicas heurísticas têm sido aplicadas com sucesso na obtenção de detectores MuD quase-ótimos, denominados detectores multiusuário heurísticos (Heur-MuD) (ERGÜN; HACIOGLU., 2000; ABRÃO et al., 2009; OLIVEIRA; CIRIACO; JESZENSKY, 2009; ABRÃO et al., 2010). Heur$\mathrm{MuD}$ são capazes de atingirem de desempenhos muito próximo aos obtidos com o $\mathrm{OMuD}$, porém com custo computacional reduzido. Estas técnicas heurísticas são baseadas na aproximação sucessiva do resultado. São gerados vetores-candidatos que são avaliados através de uma Função-Custo (fitness value) que analisa a aptidão desses candidatos em relação a solução ótima, os melhores candidatos permanecem e são comparados com candidatos de um próximo ciclo e, assim, sucessivamente. A baixa complexidade juntamente com os bons resultados obtidos através destas técnicas fazem com que os métodos heurísticos sejam muito bem aceitos e viáveis na detecção $\mathrm{MuD}$.

Dentre as técnicas heurísticas, emprega-se neste trabalho o algoritmo de busca local (LS - Local Search) (AARTS; LENSTRA, 2003; OLIVEIRA; CIRIACO; JESZENSKY, 2009), por suas características de baixíssima complexidade na resolução do problema $\mathrm{MuD}$.

Conforme foi mencionado, cada detector multiusuário apresenta suas vantagens e desvantagens. Com exceção do detector ótimo, que tem sua implementação impraticável para os padrões de tecnologia atuais, os outros detectores têm sua aplicação viável. Porém, as técnicas heurísticas se apresentam mais atrativas por combinarem diferentes aspectos que se encaixam perfeitamente às limitações de capacidade computacionais existentes atualmente. Dois fatores são essenciais na análise de viabilidade de implementação de um $\mathrm{MuD}$, são eles: desempenho e complexidade computacional (processamento).

Por terem seu processo de busca baseado na avaliação de uma função custo desenvolvida a partir do princípio OMuD, os Heur-MuD apresentam desempenhos muito próximo ao desempenho ótimo, atingido pelo OMuD. Quanto à complexidade, a aplicação de técnicas heurísticas ao problema $\mathrm{MuD}$ resulta em uma função polinomial dependente apenas de parâmetros do sistema, o que reduz significativamente o tempo de processamento. As características mais marcantes dos detectores HeurMuD são:

- complexidade reduzida em relação ao OMuD;

- desempenho muito próximo ao do $\mathrm{OMuD}$;

- tolerância ao efeito near-far e ao carregamento do sistema (ou à MAI).

Desta forma, existe grande viabilidade de implementação dos Heur-MuD através de uma plataforma de DSP, conforme proposto neste trabalho.

Uma das áreas mais dinâmicas dos sistemas digitais, hoje em dia, está no campo do processamento digital de sinais. Processadores de sinais digitais são microprocessadores rápidos feitos com um tipo especializado de arquitetura e um conjunto de instruções adequadas para o processamento de sinal.

Os processadores convencionais, ou de uso geral, são projetados para manipular dados, não 
possuindo estruturas dedicadas para processamento digital de sinais. Enquanto a grande maioria das aplicações dos processadores convencionais são off-line, as aplicações em DSP são, em sua maioria, aplicações em tempo real. Nas aplicações off-line os dados estão disponibilizados para o processamento e um pequeno atraso não prejudica o resultado do mesmo. Nas aplicações online, ou tempo real, os dados devem ser processados à medida que são recebidos e são tolerados apenas atrasos muitos pequenos.

Os DSPs podem ser empregados em diversas áreas como também para processamentos específicos, onde deseja-se encontrar soluções adaptativas para um determinado problema. A simulação em tempo real é uma área que tem sido bastante estudada ao longo dos anos, existindo diversos trabalhos de programação para controle de processos. Assim, a questão da limitação de tempo tornou-se um problema a ser solucionado, pois garantir uma resposta certa em tempo real em alto nível torna-se complexo, especialmente para DSPs onde sistemas com determinada taxas de dados devem ser analisados (MARWEDEL, 1995).

A utilização de software para simulações computacionais como o Matlab não impõe um determinado tempo para que tal simulação ocorra. Porém, ao se trabalhar com aplicações em tempo real, faz-se necessário a utilização de processadores digital de sinais que implementará tais processamentos de uma determinada amostra, antes que novos dados cheguem para serem processados.

Há outros trabalhos que fizeram uso de uma plataforma DSP para implementação de detectores multiusuários em sistemas DS/CDMA. Em (RAJAGOPAL; XU; CAVALLARO, 1999), foi feita a detecção multiusuário e estimação dos parâmetros do canal em sistemas CDMA de bandalarga (W-CDMA: Wideband-CDMA), sendo este o protocolo usado nos sistemas de comunicação de tecnologia 3G. A técnica de detecção multiusuário utilizada foi baseada no algoritmo de Diferenciação em Multiestágios (Differencing Multistage). Para a detecção foi utilizada uma plataforma DSP pontofixo de 16 bits, enquanto que, para estimação dos parâmetros de canal uma plataforma DSP de ponto-flutuante foi empregada devido à maior complexidade envolvida nas operações do estimador, como cálculos de inversas de matrizes. Um ponto bastante interessante neste trabalho são as técnicas de otimização empregadas. Além da implementação em plataforma DSP, foram realizadas diferentes métodos de otimização do código implementado em DSP, de forma que se obtivesse o menor tempo de processamento e, consequentemente, o melhor desempenho. Mostra-se que a melhor técnica de otimização deu-se trabalhando com o código assembly em regiões críticas do algoritmo, como multiplicações entre matrizes de grandes dimensões, isto resultou numa melhora bastante significativa no tempo de processamento tanto do estimador quanto do detector. Mesmo com bons métodos de otimização, mostra-se que o processamento em tempo real ainda não foi possível e os requerimentos para que possa ser atingido também são analisados pelo autor.

Em (K.WACKER et al., 2001), foi realizada uma implementação de detector multiusuário baseado na técnica PIC com estimador de canal em sistemas W-CDMA utilizando plataforma DSP ponto-fixo de 16 bits. O foco deste trabalho se deu, além da implementação, na utilização de diversos esquemas de modulação. Mostra-se que a plataforma DSP ponto-fixo é completamente capaz de suportar a implementação com perda de desempenho marginal em relação a uma plataforma ponto-flutuante. Uma conclusão importante realizada neste trabalho se dá no fato da utilização de múltiplas plataformas DSP, uma para cada usuário ativo do sistema e uma central para o processamento do PIC, trazendo um significativo aumento de desempenho ao sistema.

\section{Ferramentas de projeto}

Nesta seção são descritas as ferramentas computacionais utilizadas neste trabalho, 
necessárias tanto para a obtenção dos resultados de desempenho, quanto para a implementação em plataforma DSP dos detectores convencional e heurístico de busca local 1-ótimo (1-opt LS), definido formalmente na Seção 3.3.

\section{Matlab}

O MATLAB -- Matrix Laboratory -- é um software da The Mathworks Inc. (http://www. mathworks.com/) destinado a fazer cálculo com matrizes de dimensões elevadas de forma eficiente. Contudo, ele integra análise numérica, cálculo com matrizes, processamento de sinais e construção de gráficos em ambiente fácil de usar onde problemas e soluções são expressos somente como eles são escritos matematicamente, ao contrário da programação tradicional, tornando mais simples o seu uso, possibilitando a incorporação de rotinas e funções definidas pelo usuário além de pacotes para cálculos específicos.

\section{DSK C6713}

O DSP utilizado neste trabalho é um TMS320C6713, fabricado pela Texas Instruments' Inc. (http://www.ti.com/), fazendo parte de um kit de desenvolvimento denominado DSK C6713. O DSP está contido em uma plataforma desenvolvida pela Spectrum Digital Inc. (http://www.spectrumdigital. com/), que integra o DSP e uma série de componentes que visam otimizar sua capacidade, como um codicador/decodicador (CODEC) analógico para digital (ADC) e digital para analógico (DAC) estéreos de 16 bits, cujas freqüências de amostragem variam entre 8 e $96 \mathrm{Kbps}$, um emulador JTAG, $16 \mathrm{MB}$ de memória SDRAM e $256 \mathrm{kB}$ de memória flash. Também está disponível na plataforma uma interface chamada de EMIP (External Memory Interface), dois barramentos I2C (Inter-Integrated Circuit), dois temporizadores, uma GPIO (General Purpose Input and Output), um HPI (Host-Port Interface), e um EDMA (Enhanced Direct Memory Access) com 16 canais independentes.
O kit também inclui um software de desenvolvimento da Texas Instruments Inc. para os DSPs de sua fabricação, denominado Code Composer Studio (CCS). Sua programação é feita através da linguagem $\mathrm{C} / \mathrm{C}++$ sendo usado para implementação já que é totalmente integrado à plataforma do DSP e compatível com o simulador matemático MatLab.

\section{DSP TMS320C6713}

O TMS320C6713 (C6713) possui arquitetura de ponto flutuante e pode processar simultaneamente 8 instruções a cada ciclo de clock e tem o desempenho equivalente a 1800 MIPS. Ele pode realizar duas operações de multiplicação e acumulação (MAC) por ciclo de clock. Também dispõe de 264KB de memória interna, das quais $4 \mathrm{~KB}$ são alocadas para a memória cache tipo L1P (Level 1 Program cache) e L1D (Level 1 Data cache) e o restante é alocado para a memória chamada de L2 que compartilha o espaço de programa e de dados. Sua arquitetura, baseada em VLIW (Very-Large-Instruction-Word), está muito bem adaptada para cálculos numéricos intensivos. O C6713 é considerado, um dos mais poderosos processadores fabricados pela Texas Instruments' (CHASSAING, 2005).

\section{Code Composer Studio}

O CCS fornece um ambiente integrado de desenvolvimento (IDE - Integrated Development Environment) para incorporar as ferramentas de software. O CCS inclui ferramentas para a geração de código, como um compilador $\mathrm{C}$, um montador e um linker. Tem capacidades gráficas e suporte para depuração em tempo real, fornecendo uma ferramenta de fácil manuseio para criar e depurar programas.

O compilador C compila um programa fonte em C com a extensão ".c" para produzir uma fonte em assembly com extensão “.asm”. O assembler gera, a partir do arquivo assembly, um arquivo 
na linguagem de máquina com extensão ".obj", chamado "object file" . O linker combina "object files" e bibliotecas ("object libraries") para produzir um arquivo executável na extensão “.out”. Este arquivo representa um arquivo executável popular em sistemas baseados em Unix e adotado por vários fabricantes de processadores de sinal digital (objeto COFF - Common Object File Format). Este arquivo executável pode ser carregado e executado diretamente sobre o processador C6713 (CHASSAING, 2005).

A análise em tempo real pode ser realizada utilizando o RTDX (Real-Time Data Exchange), que é uma ferramenta do CCS. O RTDX permite a troca de dados entre o PC e a plataforma DSP através do CCS, tendo a análise avaliada em tempo real, ou seja, sem a interrupção no processamento do DSP.

\section{Real-Time Data Exchange}

O RTDX fornece, em tempo real, a visibilidade contínua nas operações realizadas numa plataforma de destino. RTDX transfere dados entre um computador, "host", e dispositivos de destino, "target", sem interferir nas aplicações realizadas no "target", ou seja, sem interrupção de processamento no "target". Você pode analisar e visualizar os dados no computador utilizando a interface fornecida pelo COM RTDX. Aplicativos-cliente, como o MATLAB, podem facilmente usar a interface COM (TEXAS INSTRUMENTS INC., 2005).

RTDX forma dois canais para caminho de dados, para envio e recepção de dados, entre um aplicativocliente no computador e a plataforma de destino através de uma combinação de componentes de hardware e software. A configuração das ligações de tráfego de dados do RTDX empregada neste trabalho é descrita na Figura (1). O host é o computador e o target é a plataforma do DSK C6713. O aplicativocliente no host é o Matlab e o dispositivo de destino no target é o processador DSP. Nesta configuração, a criação e habilitação dos canais de entrada e saída do RTDX devem ser feitas em ambos aplicativos; Matlab e CCS. Uma vez criados, os canais podem transmitir dados do aplicativo-cliente para o dispositivo de destino e vice-versa. Porém, o processador DSP deve estar rodando para que seja possível esta troca de dados em tempo-real onde não há interrupção de seu processamento. Quando um comando de recepção de dados via RTDX é executado, há uma pausa na execução do código até que um comando de escrita de dados seja executado no outro lado da comunicação RTDX.

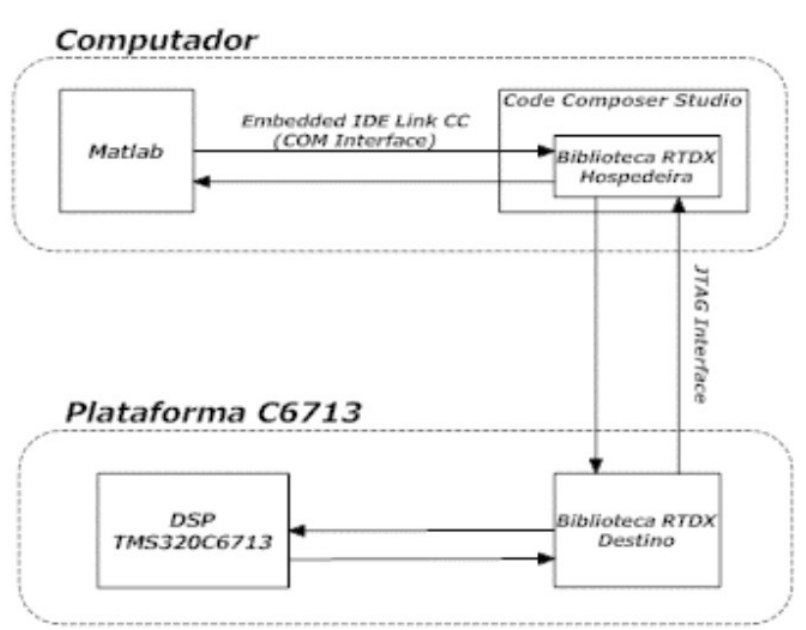

Figura 1. Configuração das ligações de tráfego de dados do RTDX empregada neste trabalho.

Alguns comandos de recepção e envio de dados via RTDX no CCS que são utilizados neste trabalho podem ser vistos na Figura (2). São exibidos os comandos para a recepção/sincronismo da sequência de espalhamento de cada usuário ativo, definida na Equação , da matriz de geração de todos os vetorescandidatos com distância de Hamming ${ }^{3}$ igual a 1, bem como do vetor contendo as amplitudes do sinal de cada usuário. Os comandos de envio exibidos referem-se, respectivamente, ao vetor-solução do 1-opt LS e ao contador de iterações do mesmo.

\footnotetext{
3 A distância de Hamming entre dois vetores é o número de bits diferentes entre eles.
} 


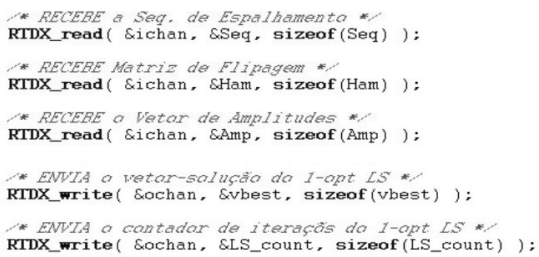

Figura 2. Alguns dos comandos de escrita e leitura de dados do canal RTDX no CCS.

Alguns comandos de recepção e envio de dados via RTDX no Matlab que são utilizados neste trabalho podem ser vistos na Figura (3). São exibidos os comandos de envio da matriz de sequências de espalhamento de cada usuário ativo, da matriz de geração dos vetores-candidatos para distância de Hamming igual a 1 e da matriz de amplitudes do sinal de cada usuário. Os comandos de recepção exibidos referem-se, respectivamente, ao vetorsolução do 1-opt LS e ao contador de iterações do mesmo realizadas no processamento em DSP.

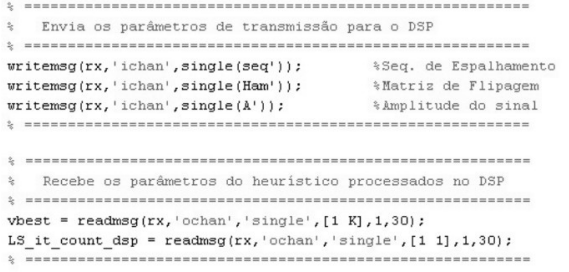

Figura 3. Alguns dos comandos de escrita e leitura de dados do canal RTDX no Matlab.

\section{Descrição do sistema de comunicação}

São descritos os três subsistemas de um DS/ CDMA: Transmissor, Receptor Convencional (CD ou $\mathrm{SuD}$ ) e o Heur-MuD usando o algoritmo de Busca Local 1-ótimo (1-opt LS - 1-Optimum Local Search). Dentre estes, o receptor 1-LS-MuD será implementado em DSP. Para todos os sistemas DS/CDMA, foi empregado um modelo de sinais em banda-base equivalente sobre um canal AWGN síncrono.

\section{Transmissor}

Este é o primeiro subsistema a ser descrito, sendo utilizado em conjunto pelo detector convencional (CD) e pelo do Heur-MuD, formando, assim, dois sistemas independentes. Este subsistema será descrito somente em linguagem Matlab.

Em um sistema DS/CDMA, com $K$ usuários ativos, utilizando modulação BPSK e canal com desvanecimento, o sinal em tempo contínuo que chega ao receptor pode ser descrito, em banda-base, como:

$$
r(t)=\sum_{k=1}^{K} A_{k} b_{k} s_{k}\left(t-\tau_{k}\right) * h(t)+\eta(t)
$$

onde $K$ é o número de usuários ativos no sistema; $t \in\left[0, T_{b}\right]$ e $T_{b}$ é o período de bit'; $A_{k}$ é a amplitude do sinal transmitido do $k$-ésimo usuário, dada por $E_{k}=P_{k} T_{b}=A_{k}^{2} T_{b}$, onde $E_{k}$ é a energia de bit e $P_{k}$ a potência do sinal recebido pelo $k$-ésimo usuário; $b_{k} \in\{-1,+1\}$ é o bit de informação transmitido do $k$-ésimo usuário, assumidos equiprováveis e independentes; $h(t)$ é a resposta impulsiva do canal e $\eta(t)$ ruído AWGN de tempo contínuo, representando o ruído térmico e outras fontes de ruído não relacionadas aos sinais transmitidos com densidade de potência bilateral igual a $N_{0} / 2$.

A sequência de espalhamento, $s_{k}$, atribuída ao $k$-ésimo usuário é dada por:

$$
s_{k}(t)=\sum_{i=0}^{N-1} z_{k}^{(i)} P_{t}\left(t-i T_{c}\right)
$$

onde $z_{k}$ é o vetor de chips, com $z_{k}^{(i)} \in\{-1,+1\}$ com duração de $T_{c} ; T_{c}$ é o intervalo de cada chip e $P_{t}(\cdot)$ é um pulso retangular de amplitude unitária no intervalo $\left[0, T_{c}\right)$.

4 Adotado sem perda de generalidade como normalizado. 
Neste trabalho, consideram-se sistemas DS/ CDMA com códigos de espalhamento curtos, isto é, o ganho de processamento é dado por $N=T_{b} / T_{c}$ - Adota-se canal AWGN síncrono $\left(\tau_{k}=0\right)$, não sendo incluído o efeito de desvanecimento de pequena escala (ruído multiplicativo), ou seja, $h(t)=\delta(t)$ na Equação . Reescrevendo a Equação e levando-se em conta os parâmetros utilizados neste trabalho, obtém-se:

$$
r(t)=\sum_{k=1}^{K} A_{k} b_{k} s_{k}(t)+\eta(t)
$$

Por ser um sistema síncrono AWGN e não seletivo em frequência, há possibilidade do uso de sequências de espalhamento do tipo Gold ou WalshHadamard (ortogonais) que têm baixas (ou nulas) propriedades de correlação cruzada reduzindo, desta forma, a interferência de múltiplo acesso. Porém, o uso destas sequências limita o número máximo de usuários ativos no sistema para um dado tamanho de sequência.

O uso de sequências de espalhamento aleatórias praticamente elimina o limite máximo de usuários ativos do sistema. Porém, não há garantia de ortogonalidade entre as sequências. Desta forma, as propriedades de correlação cruzada entre as sequências não serão geralmente baixas, consequentemente, os sinais dos usuários sofrerão uma MAI mais elevada. Contudo, este percalço não é desvantajoso neste trabalho, pois, o foco aqui é a implementação do receptor, sendo a condição de pior caso (em relação às sequências Gold ou Walsh-Hadamard) preferencial por ser mais geral e prático. Como o CD será comparado ao Heur-MuD, pode-se melhor analisar o aumento de desempenho causado pelo Heur-MuD pela eliminação da elevada MAI. Portanto, neste trabalho serão consideradas sequências de espalhamento aleatórias.

Este é o sinal em tempo contínuo que chega ao receptor, consistindo da soma de sinais antipodais espalhados por sequências de espalhamento síncronas imersas em um canal AWGN. O transmissor abordado pode ser visualizado pelo bloco (a) da Figura (4). O modelo de canal adotado, AWGN síncrono, é descrito no bloco (b) da Figura (4).

\section{Detector convencional}

Este é o segundo subsistema a ser descrito em linguagem Matlab e implementado em DSP devido a compor um estágio do detector multiusuário heurístico. Compõe com o transmissor um sistema DS/CDMA que tem por objetivo ser comparado ao 1-opt LS-MuD podendo assim ser desenvolvida uma análise de desempenho em uma base comparativa.

Para uma recepção coerente em canal AWGN síncrono, o sinal à saída de um banco de filtros casados (MFB) à sequência de espalhamento de cada usuário, ou receptor Convencional, pode ser escrito como:

$$
y_{k}=\int_{0}^{T_{b}} r(t) s_{k}(t) d t=A_{k} b_{k}+\sum_{j \neq k}^{K} A_{j} b_{j} \lambda_{k, j}+\eta_{k}
$$

onde $\eta_{k}$ é o ruído AWGN filtrado para o $k$-ésimo usuário e $\lambda_{k, j}$ denota o $k, j$-ésimo elemento da matriz de correlação $\mathbf{R}$.

O conjunto de sinais da Equação $(k=1,2, \ldots K)$ pode ser expressa na forma matricial:

$$
\mathbf{y}=\mathbf{R A b}+\eta
$$

Sendo $\mathbf{y}$ um vetor $K \times 1, \quad \mathbf{R}_{\mathbf{K x K}}$ : matriz de correlação, A é a diagonal principal da matriz de amplitudes e b é o vetor contendo um bit de informação de cada usuário.

Para sinalização BPSK, o CD toma decisões baseado na polaridade dos sinais à saída do integrador, $\mathbf{y}$, obtendo o vetor de informação estimada:

\footnotetext{
5 Decisor abrupto (hard).
} 


$$
\hat{\mathbf{b}}=\operatorname{sgn}[\mathbf{y}]=\left\{\begin{array}{l}
-1: \mathbf{y}<0 \\
+1: \mathbf{y} \geq 0
\end{array}\right.
$$

A função sinal, $\operatorname{sgn}[\cdot]$, retorna a polaridade de um número real.

O subsistema de detecção convencional (CD) adotado pode ser visualizado por meio dos blocos (c) e (d) da Figura (4). Neste sistema de detecção, fica claro a limitação de desempenho devido à interferência de múltiplo acesso, uma vez que o sinal a ser transmitido de cada usuário é somado aos demais usuários interferentes (termo $\sum_{j \neq k}^{K} A_{j} b_{j} \lambda_{k, j}$ da Equação) e no CD não há uma estratégia que considere as informações dos interferentes no processo de detecção do usuário de interesse.

\section{Detector multiusuário baseado no algoritmo} heurístico de busca local

Este é o terceiro subsistema a ser descrito em linguagem Matlab e implementado efetivamente empregando-se a plataforma do DSK C6713.

O algoritmo heurístico utilizado neste trabalho foi o de busca local (LS). O algoritmo LS é um método de otimização que se caracteriza pela varredura em uma vizinhança pré-estabelecida do universo de busca (AARTS; LENSTRA, 2003). Nesse método, é importante a escolha da solução inicial e a limitação da vizinhança para se encontrar uma solução válida com uma complexidade computacional aceitável. Devido a sua simplicidade, o algoritmo de busca local não apresenta estratégias de escapes de máximos ou mínimos locais.

Uma outra característica dos algoritmos de busca local é a existência de duas estratégias para a realização do deslocamento no espaço de busca. Existe a estratégia de deslocamento pelo maior ganho e a estratégia de deslocamento pelo primeiro ganho. A primeira utiliza a melhor solução de uma vizinhança como entrada da próxima iteração. A outra estratégia utiliza a primeira solução que apresentar um maior valor que a solução atual como entrada da próxima iteração (CIRIACO, 2004). Neste trabalho, utilizou-se a estratégia de deslocamento pelo maior ganho.

No intuito de diminuir a complexidade computacional e, consequentemente, o tempo computacional, foi feita a escolha do algoritmo de busca local 1-ótimo (1-opt LS). Este algoritmo procura por uma solução ao redor de uma vizinhança composta por todas as possíveis soluções cuja distância de Hamming, Equação, é igual a 1. A cada nova iteração de busca no LS, todos os vetores com distância de Hamming igual a 1, chamados de vetores-candidatos $(\vartheta)$, são avaliados através do cômputo da Função-Custo ( $F$ ), ver Equação . O melhor vetor obtido através da avaliação é então adotado como sendo o novo ponto base de cálculo da vizinhança para a próxima iteração.

$$
V\left(\vartheta^{m}\right)=\left\{\vartheta_{i} \in\{-1,1\}^{K} \text { tal que }\left\|\vartheta_{i}-\vartheta_{m}\right\|=1\right\}
$$

onde $\vartheta^{m}$ é o vetor-base; $M_{t}$ é o número máximo de iterações e $m=1, \ldots, M_{t}$ e $i=1, \ldots, K$.

O vetor-candidato inicial tomado como base $\left(\vartheta_{\text {inicial }}^{\text {best }}\right.$ ) para cálculo da vizinhança com distância de Hamming igual a 1 é dado pela saída do CD. Neste trabalho, um detector convencional abrupto (decisão $h a r d$ ). Os vetores-candidatos são obtidos conforme a Equação . Como o vetor à saída do $\mathrm{CD}$ tem $K$ bits ( $K=$ número de usuários) e a distância de Hamming é igual a 1 são gerados vetorescandidatos em número equivalente ao número de bits do vetor-base. Portanto, serão gerados $K$ vetorescandidatos, ou seja, há $K$ possíveis soluções.

Todos vetores-candidatos têm sua aptidão avaliada por uma Função-Custo que é baseada (tomada a parte real) na função de verossimilhança (ML):

$$
F(\vartheta)=\operatorname{Re}\left\{2 \mathbf{y}^{T} \mathbf{C}^{H} \mathbf{A} \vartheta-\vartheta^{T} \mathbf{C A R A} \mathbf{C}^{H} \vartheta\right\}
$$


onde $\mathbf{y}$ é o vetor de saída do $1^{\circ}$ estágio do MFB (antes do decisor abrupto); Cé a matriz diagonal dos coeficientes do canal; A é a matriz de amplitudes dos sinais dos usuários (ganho) e $\mathbf{R}$ é a matriz de correlação.

Neste trabalho, não foi considerado um ruído multiplicativo. Portanto, pode-se definir a matriz diagonal dos coeficientes do canal igual a $1(\mathbf{C}=1)$. Desta forma, a Equação pode ser simplificada, sendo reescrita como:

$$
F(\vartheta)=\operatorname{Re}\left\{2 \mathbf{y}^{T} \mathbf{A} \vartheta-\vartheta^{T} \mathbf{A} \mathbf{R A} \vartheta\right\}
$$

O algoritmo LS tem como objetivo a maximização da Função-Custo através de possíveis soluções $(\vartheta)$ e tem um desempenho que se aproxima do detector ótimo $\mathrm{OMuD}$ à medida que o número de iterações $(m)$ aumenta. Neste trabalho, foi adotada uma estratégia com o intuito de se minimizar a complexidade envolvida no algoritmo LS. A função-custo global é dada por $F^{\text {best }}$ e representa a melhor solução global entre todas iterações. Ao invés de se fixar o número de iterações a serem realizadas pelo algoritmo, é adotado um método em que não havendo melhora da função-custo global em relação às avaliações de aptidão dos vetores-candidatos da iteração atual, o algoritmo é finalizado não existindo próxima iteração.

No diagrama de blocos da Figura (4), o algoritmo LS é representado pelo bloco (e). Desta forma, o 1-LS-MuD é constituído pelo conjunto dos blocos (c) e (d), que formam o detector convencional, juntamente com o bloco (e) representando a estratégia heurística na detecção multiusuário DS/ CDMA.

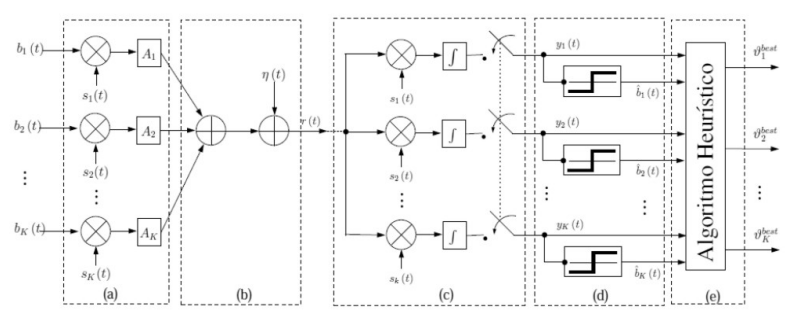

Figura 4. Sistema Transmissão-Recepção 1-LS-MuD - (a) Transmissor em banda-base; (b) Modelo de canal adotado; (c) $1^{\circ}$ estágio MFB; (d) Decisor Abrupto com ponto de derivação das estimativas do MFB e (e) Algoritmo Heurístico 1-opt LS.

\section{Metodologia}

Esta seção descreve a metodologia empregada na elaboração, implementação em DSP e caracterização dos subsistemas DS/CDMA.

Todas as simulações e implementações realizadas neste trabalho são baseadas no Método de Simulação Monte Carlo (MCS - Monte Carlo Simulation). As estimativas da quantidade de trials a serem transmitidos, ou seja, o número de ciclos (cada ciclo transmite um bit de cada usuário), foi feita considerando o número de erros, $\mathrm{n}_{\text {erros }}$, estimados no Single-User Bound (SuB), pois este indica o melhor desempenho possível do sistema na condição de ausência de interferência de múltiplo acesso (um único usuário ativo). Considera-se $\mathrm{K}$ como o número de usuários e $\mathrm{N}$ o ganho de processamento.

A Fig. 5 mostra um esquema simplificado das implementações dos sistemas DS/CDMA realizadas. Como foi explicado na Seção 3, o transmissor em banda base será somente descrito em linguagem Matlab, enquanto que o detector multiusuário heurístico baseado no algoritmo de busca local 1-ótimo será tanto implementado através da plataforma C6713 quanto descrito em linguagem Matlab. Apesar do CD ser implementado em DSP como parte do $1-L S-M u D$, seu vetor de saída contendo um bit de informação estimada de cada usuário não será enviado ao Matlab para análise dos resultados 
devido ao enfoque do trabalho estar voltado para implementação do detector multiusuário heurístico. Desta forma, somente o vetor solução à saída do 1-LS-MuD, resultante da implementação em DSP, será enviado ao Matlab para geração de suas figuras de mérito. A análise de complexidade realizada é descrita no artigo (RIBEIRO, 2009).

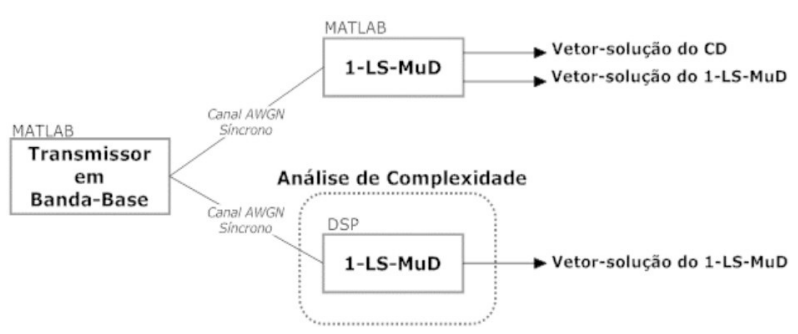

Figura 5. Esquema geral simplificado das implementações realizadas.

\section{Transmissor 1-LS-MuD em banda base para} sistema DS/CDMA

Este sistema terá duas abordagens diferentes: o primeiro será descrito inteiramente em linguagem MATLAB para servir de base comparativa. Porém, o enfoque nesta seção será dado ao segundo sistema que será parte em MATLAB e parte em DSP via CCS. Na implementação feita em CCS, para ser processada no DSP, emprega-se a linguagem C. Foram criadas funções específicas para adição, multiplicação de matrizes, como também, uma função com o algoritmo 1-opt LS. Todas estas funções foram armazenadas em uma biblioteca que é adicionada ao projeto do CCS.

A Fig. 6 ilustra um diagrama de blocos do sistema DS/CDMA desenvolvido através da comunicação RTDX entre MATLAB e DSP. A análise de complexidade computacional envolvida na implementação do $1-L S-M u D$ é apresentada no artigo (RIBEIRO, 2009).

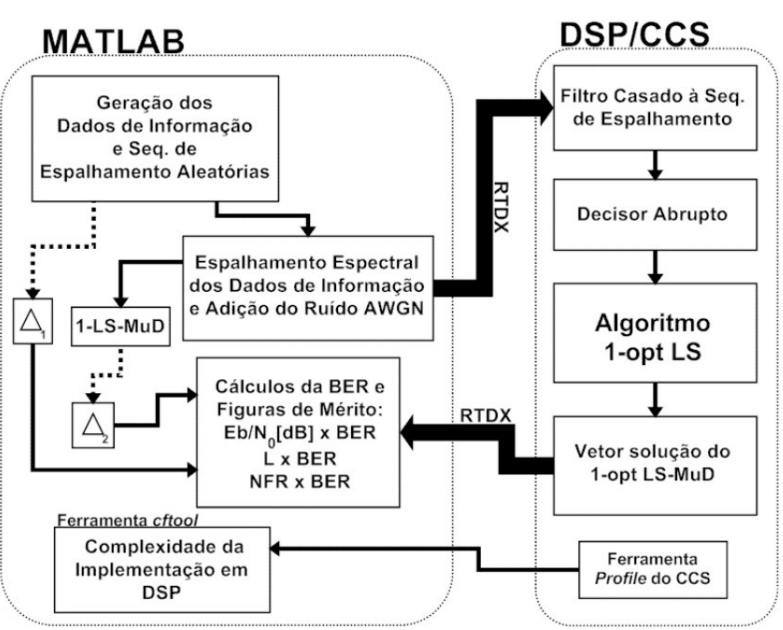

Figura 6. Diagrama de blocos do sistema DS/CDMA com LS-MuD implementado em MATLAB e DSP.

O fluxograma da Fig. 7 descreve detalhadamente cada passo desenvolvido na implementação em DSP do sistema Transmissor 1-opt LS-MuD. Neste fluxograma é incluído o bloco de detecção 1-opt $L S-M u D$ realizado em Matlab para servir de base comparativa junto ao vetor-solução do mesmo detector processado em DSP. A subrotina de Transmissão-Recepção que denota cada trial processado é ilustrada na Fig. 8, nela os processos marcados com (DSP) ao fim da descrição são blocos processados através da plataforma DSP.

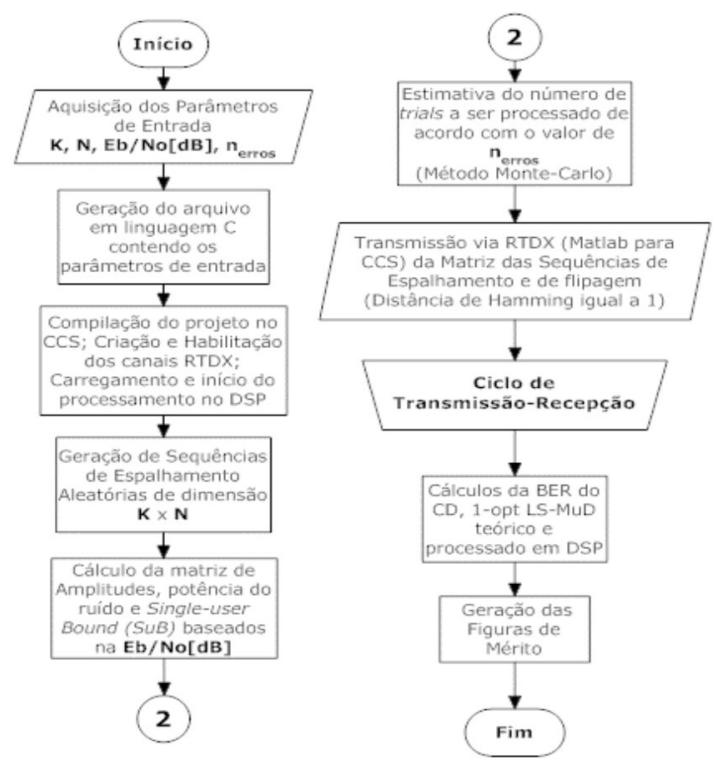

Figura 7. Fluxograma descrevendo a metodologia empregada na implementação em DSP do sistema DS/ CDMA entre Transmissor e 1-LS-MuD. 


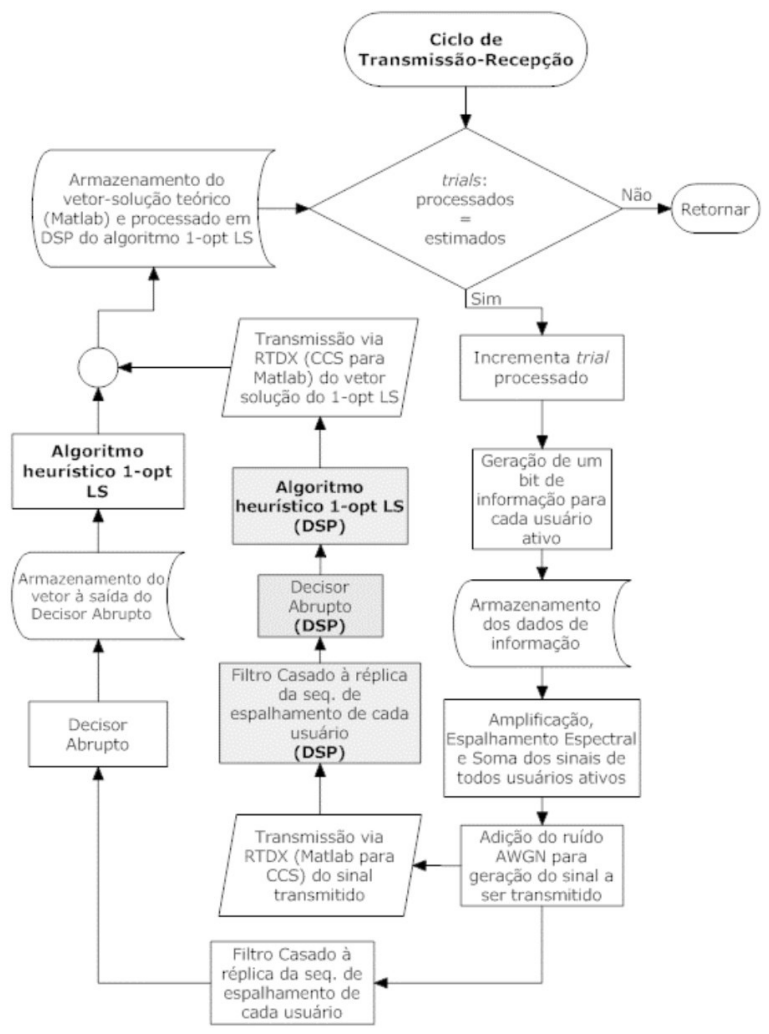

Figura 8. Fluxograma descrevendo a implementação em DSP da sub-rotina de Transmissão-Recepção Multiusuário Heurística do sistema DS/CDMA.

\section{Resultados}

Nesta seção serão apresentados os resultados em DSP. Os parâmetros dos processamentos escolhidos visam validar o uso do DSP na implementação de um receptor heurístico utilizando o algoritmo 1-opt $L S$ em sistemas DS/CDMA. Foram feitas duas abordagens, totalizando 3 implementações:

- Avaliação de Desempenho através da Variação da SNR (Signal-to-Noise Ratio);

- Robustez do Sistema, composta por duas implementações:

- Variação do Índice de Carregamento;

- Near-far Ratio.

As 3 implementações serão avaliadas em relação a BER (Bit Error Rate).
Desempenho do sistema - variação da SNR

Foram feitos dois processamento para avaliação do desempenho do sistema: em baixo e em alto carregamento.

No processamento de baixo carregamento, foram considerados um ganho de processamento igual a $15(\mathrm{~N}=15)$ e o número de usuários ativos de $5(\mathrm{~K}$ $=5$ ). Portanto, assume-se um carregamento de 0,33 $(\mathrm{K} / \mathrm{N})$. A variação da $\mathrm{E}_{\mathrm{b}} / \mathrm{N}_{0}[\mathrm{~dB}]$ foi de $0 \mathrm{~dB}$ até $8 \mathrm{~dB}$ com passos de $2 \mathrm{~dB}$ e o número de erros em cada ponto é mostrado nos parâmetros de entrada. Os parâmetros de entrada deste processamento podem ser vistos na Fig. 9 e os resultados na Fig. 10.

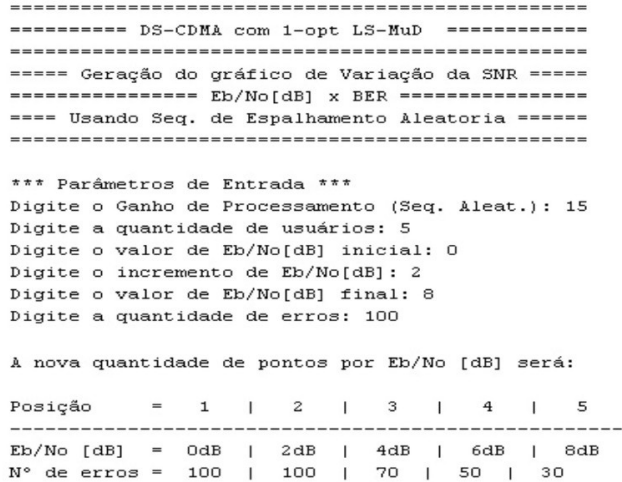

Figura 9. Parâmetros de entrada utilizados no processamento em DSP para avaliação de desempenho com baixo carregamento.

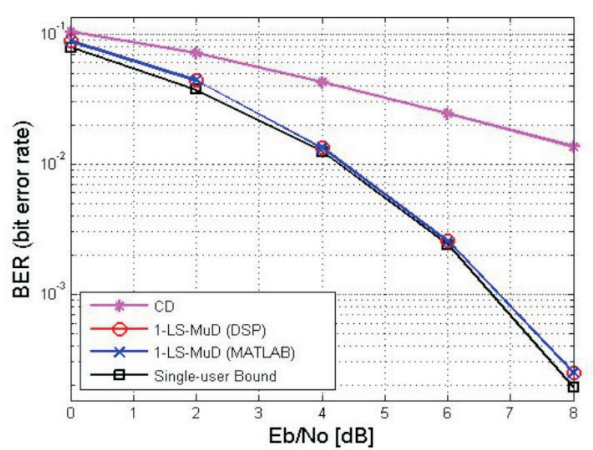

Figura 10. Resultados do processamento em DSP para avaliação de desempenho com baixo carregamento do sistema transmissor/receptor 1-opt LS-MuD implementado para $\mathrm{K}=5, \mathrm{~N}=15$ e $\mathrm{L}=0,33$. 
No processamento de alto carregamento, foram considerados um ganho de processamento igual a $15(\mathrm{~N}=15)$ e o número de usuários ativos de $12(\mathrm{~K}$ =12). Portanto, assume-se um carregamento de 0,8 $(\mathrm{K} / \mathrm{N})$. A variação da $\mathrm{E}_{\mathrm{b}} / \mathrm{N}_{0}[\mathrm{~dB}]$ foi de $0 \mathrm{~dB}$ até $8 \mathrm{~dB}$ com passos de $2 \mathrm{~dB}$ e o número de erros em cada ponto é mostrado nos parâmetros de entrada. Os parâmetros de entrada deste processamento podem ser vistos na Fig. 11 e os resultados na Fig. 12.

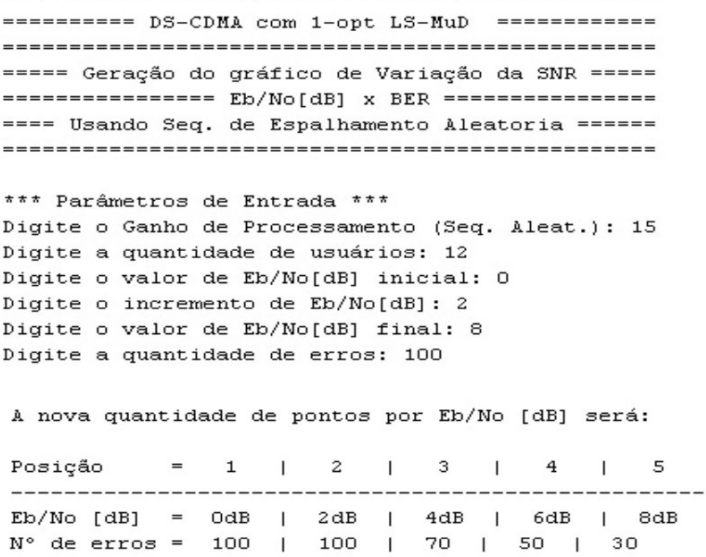

Figura 11. Parâmetros de entrada utilizados no processamento em DSP para avaliação de desempenho com alto carregamento.

Os resultados do processamento em DSP foram equivalentes aos obtidos via simulação computacional em Matlab, demonstrando a viabilidade da implementação deste sistema em plataformas DSPs. Além disso, observa-se que a técnica heurística aplicada ao problema $\mathrm{MuD}$ resultou em desempenhos próximos ao ótimo sob todas as condições de operação de sistema e de canal AWGN analisad

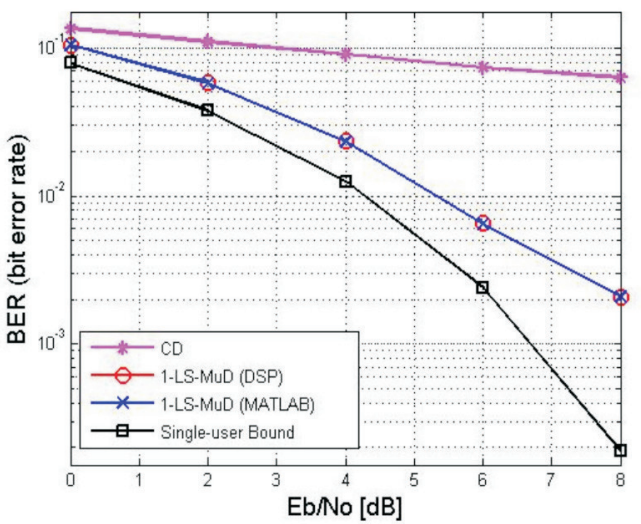

Figura 12. Resultados do processamento em DSP para avaliação de desempenho com alto carregamento do sistema transmissor/receptor 1-opt LS-MuD implementado para $\mathrm{K}=12, \mathrm{~N}=15$ e $\mathrm{L}=0,8$.

\section{Robustez do sistema}

A robustez do sistema pode ser avaliada através de dois processamentos em DSP: Carregamento do Sistema e NFR (Near-far Ratio).

\section{Carregamento do Sistema}

Para o processamento em DSP do Carregamento do Sistema, considerando as observações feitas no início deste capítulo, houve um ajuste do número de usuários e ganho de processamento, de forma que, em cada ponto pudesse ser explorado o limiar de 12 usuários. O número de erros em cada ponto foi decidido de forma inversamente proporcional ao ganho de processamento, sendo que o valor de 30 erros foi escolhido inicialmente para ganho de processamento igual a 30 . A quantidade de usuários e o ganho de processamento podem ser vistos na Tabela 1. A $\mathrm{E}_{\mathrm{B}} / \mathrm{N}_{0}[\mathrm{~dB}]$ considerada foi de $7 \mathrm{~dB}$, de forma que o valor do limite $S u B$ estivesse num patamar mínimo de $10^{-3}$. 
Tabela 1 - Número de Usuários (K) e Ganho de Processamento (N) em cada Índice de Carregamento (L) do Sistema.

\begin{tabular}{ccc}
\hline $\begin{array}{c}\text { Índice de } \\
\text { Carregamento } \\
(\mathrm{L})\end{array}$ & $\begin{array}{c}\text { Usuários } \\
\text { Ativos }(\mathrm{K})\end{array}$ & $\begin{array}{c}\text { Ganho de } \\
\text { Processamento } \\
(\mathrm{N})\end{array}$ \\
\hline 0,10 & 3 & 30 \\
0,20 & 6 & 30 \\
0,30 & 9 & 30 \\
0,40 & 12 & 30 \\
0,50 & 10 & 20 \\
0,60 & 12 & 20 \\
0,67 & 10 & 15 \\
0,80 & 12 & 15 \\
0,92 & 11 & 12 \\
1,00 & 12 & 12 \\
\hline
\end{tabular}

Os parâmetros de entrada deste processamento podem ser visto na Fig. 13 e os resultados na Fig. 14.

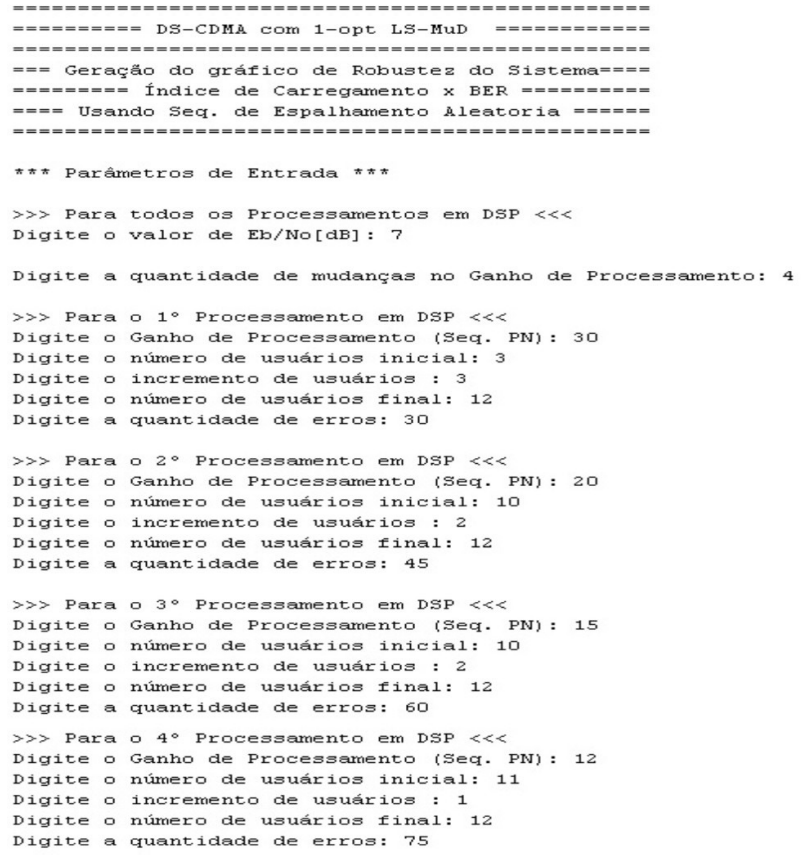

Figura 13. Parâmetros de entrada utilizados no processamento em DSP do Carregamento do Sistema transmissor/receptor 1-opt LS-MuD implementado.

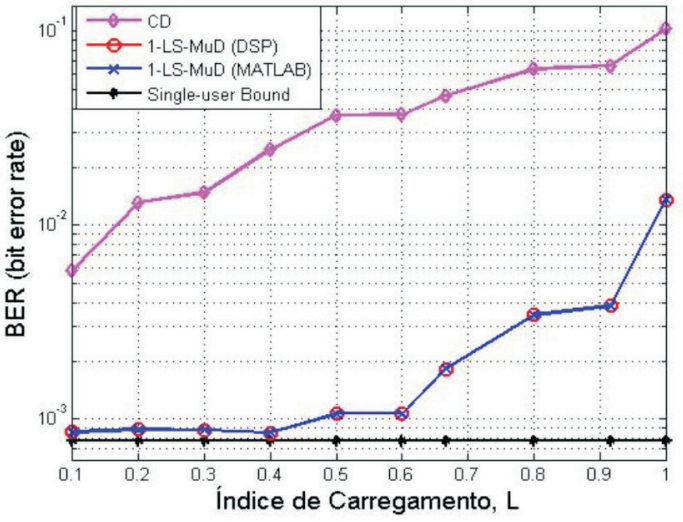

Figura 14. Resultados do processamento em DSP do Carregamento do Sistema transmissor/receptor 1-opt LS$\mathrm{MuD}$ implementado para $\mathrm{E}_{\mathrm{b}} / \mathrm{N}_{0}[\mathrm{~dB}]=7 \mathrm{~dB}$ e $\mathrm{K}$ e $\mathrm{N}$ de acordo com a Tabela 1 .

\section{Near-Far Ratio}

Este processamento em DSP vem validar a tolerância do sistema ao efeito near-far, chamada de NFR. Este efeito acontece quando os usuários de interesse mantém-se com o valor da $\mathrm{E}_{\mathrm{b}} / \mathrm{N}_{0}[\mathrm{~dB}]$ fixa (distância fixa em relação a ERB) enquanto que os restantes dos usuários ativos do sistema sofrem variação da sua $\mathrm{E}_{\mathrm{b}} / \mathrm{N}_{0}[\mathrm{~dB}]$ (movem-se em relação a ERB). O cálculo da BER é feito somente para os usuários de interesse e, apesar de ativos, o restante dos usuários são considerados interferentes e não entram no cômputo da BER.

No processamento em DSP foi considerado ganho de processamento igual a 15, 4 usuários de interesse, 8 usuários interferentes, $\mathrm{E}_{\mathrm{b}} / \mathrm{N}_{0}[\mathrm{~dB}]$ do usuário de interesse fixa em $7 \mathrm{~dB}$ e $\mathrm{E}_{\mathrm{b}} / \mathrm{N}_{0}[\mathrm{~dB}]$ dos usuários interferentes variando de 1 a $13 \mathrm{~dB}$ com passos de $2 \mathrm{~dB}$, ou seja, uma variação de -6 a $6 \mathrm{~dB}$ em relação aos usuários de interesse. Os parâmetros de entrada deste processamento podem ser vistos na Fig. 15 e os resultados de desempenho na Fig. 16. 


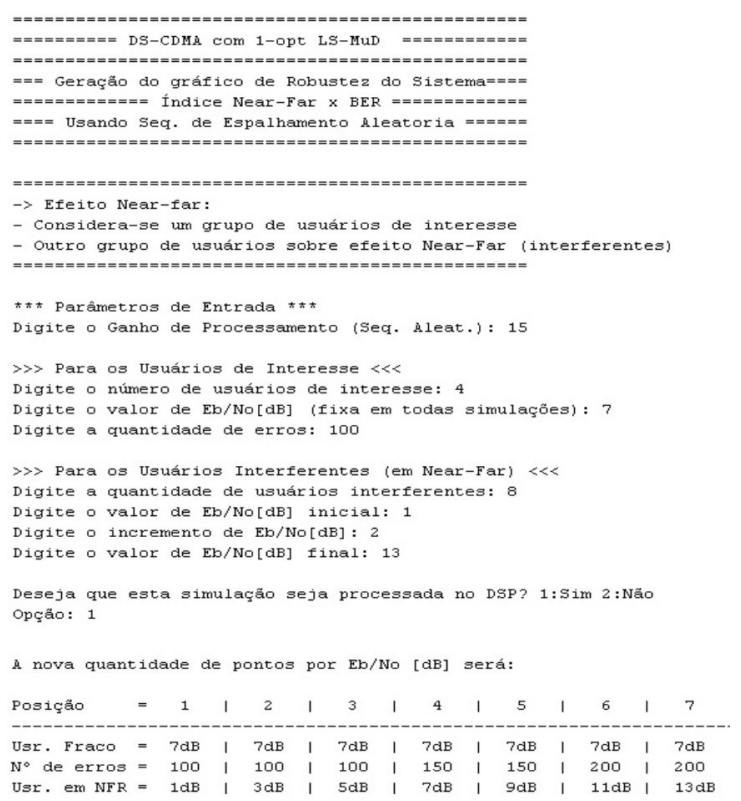

Figura 15. Parâmetros de entrada utilizados no processamento em DSP para tolerância ao Efeito NearFar no Sistema transmissor/receptor 1-opt LS-MuD implementado.

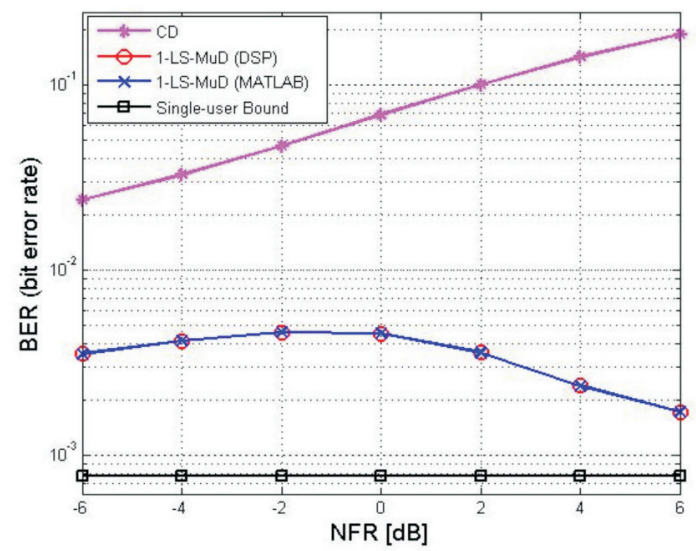

Figura 16. Resultados do processamento em DSP para tolerância ao efeito Near-Far no Sistema Tx/Rx 1-opt LS-MuD implementado para $\mathrm{N}=15, \mathrm{~K}_{\text {desejado }}=4, \mathrm{~K}_{\text {interf }}=8$ e $\mathrm{E}_{\mathrm{b}} / \mathrm{N}_{0}[\mathrm{~dB}]$ dos usuários de interesse igual a $7 \mathrm{~dB}$.

Os resultados do processamento em DSP foram novamente equivalentes aos obtidos via simulação em Matlab. Adicionalmente, observa-se que a técnica heurística aplicada a este problema demonstrou uma boa robustez ao carregamento do sistema e ao efeito Near-Far.

Percebe-se que todos os resultados de processamento em DSP exibidos neste capitulo foram coincidentes com os da simulação descrita em linguagem Matlab. Isto se deve ao fato de que o código em linguagem $\mathrm{C}$ executa as mesmas operações que a simulação feita em Matlab e, como não há nenhum dado aleatório sendo gerado no processamento em DSP, não há variações nos resultados encontrados. Um outro fator que contribui para a semelhança nos resultados é que o DSP possui uma estrutura computacional bastante poderosa sendo dedicado a cálculos matemáticos de adição e multiplicação e ainda possui estratégias para a minimização dos erros de quantização, desta forma, sua performance é bastante superior se comparada a outros microcontroladores como Motorola MC68HC16 e Intel 80196.

\section{Conclusões}

Um sistema de comunicação DS/CDMA em banda base foi implementado em DSP comercial; os processamentos de sinais correspondentes foram previamente emulados através do simulador matemático Matlab. Apesar das limitações em relação ao número de usuários ativos, pôde-se efetuar os principais testes que validam a funcionalidade do sistema DS/CDMA implementado dentro das condições de canal estabelecidas.

Um grande obstáculo enfrentado durante a implementação em DSP se deu em relação as operações matriciais com números reais e complexos. Não foi encontrada uma biblioteca adequada que servisse para as operações necessárias na recepção heurística do sistema DS/CDMA abordado. Desta forma, fez-se necessário o desenvolvimento de uma biblioteca própria com praticamente todas operações matriciais utilizadas no projeto em CCS. Na manipulação de números complexos, foi seguido o mesmo procedimento. Após a escolha de uma forma de declaração e atribuição de números complexos, todas operações associadas foram executadas através do uso de funções contidas em um arquivo em linguagem $\mathrm{C}$ adicionado ao projeto, como biblioteca. 
Para a maioria das aplicações em sistemas de comunicação faz-se necessário atender requisitos mínimos de qualidade de serviço, especialmente o requisito taxa de transferência mínima de bits (taxa de informação), relativa ao tipo de aplicação oferecida, por exemplo, serviços de voz, dados, videoconferência etc. Apesar de se tratar de um dos mais poderosos processadores de uso geral comercial da Texas Instruments Inc., os processamentos em DSP foram mais lentos que as simulações realizadas em Matlab. Portanto, avaliando-se aplicações em tempo real, o uso da plataforma DSP utilizada apresenta uma baixa aplicabilidade para serviços como de voz (MUSSI, 2009). Com uma otimização do código implementado, como através de ferramentas disponíveis no próprio CCS ou técnicas de otimização em regiões críticas (multiplicações matriciais de grandes dimensões, por exemplo) do código, há possibilidade de redução nos tempos de processamento em DSP, permitindo conjecturar sobre um aumento do número máximo de usuários de voz que podem ser processados simultaneamente pelo DSP, tanto empregando algoritmo para o receptor convencional quanto para o receptor multiusuário heurístico.

\section{Referências}

AARTS, E.; LENSTRA, J. K. Local search in combinatorial optimization. Princeton: Princeton University Press, 2003.

ABRÃO, T. Canceladores de inteferência multiusuário aplicados a sistemas DS/CDMA de múltipla taxa. 2001. Tese (Doutorado em Sistemas Eletrônicos) — Escola Politécnica da Universidade de São Paulo, São Paulo.

ABRÃO, T.; OLIVEIRA, L. D.; CIRIACO, F.; ANGÉLICO, B. A.; JESZENSKY, P. J. E. PSO assisted multiuser detection for DS-CDMA communication systems. In: OLSSON, A. E. (Org.). Particle swarm optimization: theory, techniques and applications. New York: Nova Science Publishers, 2010. v. 1, p. 247-278.

ABRÃO, T.; OLIVEIRA, L. D.; CIRIACO, F.; EVANGÉLICO, B. A.; JESZENKY, P. J. E.; CASADEVALL, F. J. P. S/Mimo MC-CDMA heuristic multiuser detectors based on single-objective optimization. Wireless Personal Communications, Dordrecht, Netherlands, v. 53, n. 4, p. 529-553, 2009.
CHASSAING, R. Digital signal processor and applications with the C6713 and C6416 DSK. New Jersey: John Wiley \& Sons Inc., 2005.

CIRIACO, F. Abordagens heurísticas aplicadas á detecção multiusuário em sistemas DS/CDMA. 2004. Tcc (Graduação em Engenharia Elétrica) - Universidade Estadual de Londrina, Londrina.

ERGÜN, C.; HACIOGLU, K. Multiuser detection using a genetic algorithm in CDMA communications systems. IEEE Transactions on Communications, New York, v. 48, n. 8, p. 1374-1382, 2000.

K.WACKER; N. G. W.; DARWOOD, P.; ALEXANDER, P.; OPPERMANN, I. Fixed point DSP implementation of iterative multi-user detection with channel estimation for UMTS. Camberra: Southern Poro Communications, 2001.

MARWEDEL, P. Code generation for embedded processors. Germany: Kluwer Academic Publishers, 1995.

MUSSI, A. M. Implementação de subsistemas DS/CDMA utilizando plataforma DSP com abordagem heurística. 2009. TCC (Graduação em Engenharia Elétrica) Universidade Estadual de Londrina, Londrina.

OLIVEIRA, L. D.; CIRIACO, F.; JESZENSKY, P. J. E. Local search multiuser detection. AEÜ. International Journal of Electronics and Communications, Wiesbaden, v. 63, n. 8, p. 259-270, 2009.

RAJAGOPAL， S.; XU, G.; CAVALLARO, J. Implementation of channel estimation and multiuser detection algorithms for W-CDMA on digital signal processors. Rice University, Houston, n. 8, p. 259-270, 1999.

RIBEIRO, R. O. Aplicabilidade de heurísticas na otimização de problemas combinatórios em telecomunicações. 2009. TCC (Graduação em Engenharia Elétrica) - Universidade Estadual de Londrina, Londrina.

TEXAS INSTRUMENTS INC. Code composer studio tutorial. Austin: Texas Instruments Inc, 2005.

VERDÚ, S. Multiuser detection. Cambridge: Cambridge University Press, 1998.

Optimum multiuser asymptotic efficiency. IEEE Transactions on Communications, New York, v. 34, n. 9 , p. $890-897,1986$.

Recebido em 12 Maio, 2010 - Received on May 12, 2010.

Aceito em 25 Outubro, 2010 - Accepted on October 25, 2010. 